\title{
ADAM12 is a circulating marker for stromal activation in pancreatic cancer and predicts response to chemotherapy
}

\author{
V. L. Veenstra ${ }^{1,2}$, H. Damhofer ${ }^{1,10}$, C. Waasdorp ${ }^{1,2}$, L. B. van Rijssen ${ }^{3}$, M. J. van de Vijver ${ }^{4}$, F. Dijk ${ }^{4}$, H. W. Wilmink,

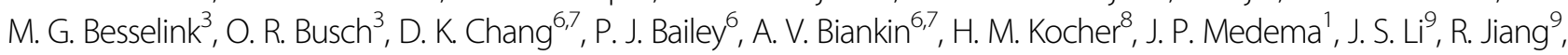 \\ D. W. Pierce ${ }^{9}$, H. W. M. van Laarhoven ${ }^{5}$ and M. F. Bijlsma ${ }^{1}$
}

\begin{abstract}
Pancreatic ductal adenocarcinoma (PDAC) is characterized by abundant stroma that harbors tumor-promoting properties. No good biomarkers exist to monitor the effect of stromal targeting therapies or to predict response. We set out to identify such non-invasive markers for PDAC stroma and predict response to therapy. Gene expression datasets, co-culture experiments, xenografts, and patient samples were analyzed. Serum samples were measured from a cohort of 58 resected patients, and 87 metastatic or locally advanced PDAC patients. Baseline and follow-up levels were assessed in 372 additional metastatic PDAC patients who received nab-paclitaxel with gemcitabine $(n=184)$ or gemcitabine monotherapy $(n=188)$ in the phase III MPACT trial. Increased levels of ADAM12 were found in PDAC patients compared to healthy controls ( $p<0.0001, n=157$ and $n=38$ ). High levels of ADAM12 significantly associated with poor outcome in resected PDAC (HR 2.07, $p=0.04$ ). In the MPACT trial survival was significantly longer for patients who received nab-paclitaxel and had undetectable ADAM12 levels before treatment (OS $12.3 \mathrm{~m}$ vs $7.9 \mathrm{~m} p=$ 0.0046). Consistently undetectable or decreased ADAM12 levels during treatment significantly associated with longer survival as well (OS $14.4 \mathrm{~m}$ and $11.2 \mathrm{~m}$, respectively vs $8.3, p=0.0054$ ). We conclude that ADAM12 is a blood-borne proxy for stromal activation, the levels of which have prognostic significance and correlate with treatment benefit.
\end{abstract}

\section{Introduction}

Survival of patients diagnosed with pancreatic ductal adenocarcinoma (PDAC) has remained poor for many decades $^{1}$. Median survival after diagnosis is around 6 months, and even patients treated with a combination of surgery and chemotherapy typically succumb to the disease within 5 years ${ }^{2}$. The vast majority of patients are diagnosed with advanced disease, precluding resection of the tumor. Treatment options for these patients are limited and clinical trials typically demonstrate only partial

\footnotetext{
Correspondence: M. F. Bijlsma (m.f.bijlsma@amc.uva.nl)

${ }^{1}$ Amsterdam UMC, University of Amsterdam, LEXOR, Center for Experimental and Molecular Medicine, Cancer Center Amsterdam, Amsterdam, Netherlands ${ }^{2}$ Oncode Institute, Meibergdreef 9, 1105AZ Amsterdam, Netherlands

Full list of author information is available at the end of the article.

Equally contributing first authors: $\mathrm{V}$. L. Veenstra, $\mathrm{H}$. Damhofer

Equally contributing senior authors: H. W. M. van Laarhoven, M. F. Bijlsma
}

benefit, in a limited number of patients. Biomarkers that allow patient stratification and early response monitoring are expected to improve outcome.

In recent years, research on PDAC has shifted focus from tumor cells to the abundant non-malignant compartment known as the stroma. This stroma consists of extracellular matrix proteins, cancer- associated fibroblasts (CAFs), and immune cells ${ }^{3}$. The stroma typically forms the vast majority of tumor bulk and contributes to chemoresistance by acting as a barrier to the delivery of chemotherapeutics. Non-mechanical tumor promoting properties have been attributed to the stroma as well ${ }^{4}$.

Nab-paclitaxel is an albumin-bound nanoparticle formulation of paclitaxel that enhances distribution and penetration into tumor tissues ${ }^{5,6}$. While the initially suspected role for SPARC (which is expressed on stromal

\section{(c) The Author(s) 2018}

(c) (i) Open Access This article is licensed under a Creative Commons Attribution 4.0 International License, which permits use, sharing, adaptation, distribution and reproduction c. in any medium or format, as long as you give appropriate credit to the original author(s) and the source, provide a link to the Creative Commons license, and indicate if changes were made. The images or other third party material in this article are included in the article's Creative Commons license, unless indicated otherwise in a credit line to the material. If material is not included in the article's Creative Commons license and your intended use is not permitted by statutory regulation or exceeds the permitted use, you will need to obtain permission directly from the copyright holder. To view a copy of this license, visit http://creativecommons.org/licenses/by/4.0/. 
fibroblasts and binds albumin) in conferring enhanced sensitivity to nab-paclitaxel in PDAC was not confirmed $^{7,8}$. The combination of nab-paclitaxel with gemcitabine is effective compared to gemcitabine monotherapy and median survival increases from 6.6 to 8.7 months $^{9}$. Other stroma targeting agents have also been tested in trials, but these have failed to demonstrate favorable outcomes ${ }^{10}$. Given the practical hurdles to obtaining tissue from the pancreas for histopathological assessment, good non-invasive markers are urgently needed that inform on the PDAC stroma to allow selection of patients for stroma-targeting therapies, and/or to monitor the response to such regimens. Currently, there is a paucity of such markers ${ }^{11}$.

ADAM12 is a member of the A Disintegrin And Metalloproteases (ADAM) protein family and harbors extracellular metalloprotease, and intracellular signaling properties. The protein is involved in cell adhesion by binding to integrins and syndecans, as well as the proteolytic cleavage of substrates from producing cells, a process known as ectodomain shedding ${ }^{12}$. Cancer-related substrates of ADAM12 include epidermal growth factor (EGF), and Sonic Hedgehog $(\mathrm{SHH})^{13-15}$. Expression of ADAM12 is elevated in glioblastoma, breast, bladder, lung, prostate, and liver cancer ${ }^{16-21}$. ADAM12 expression correlates with tumor stage in breast and bladder cancer, and is prognostic in small cell lung cancer ${ }^{16,21,22}$. A previous study showed the upregulation of ADAM12 in pancreatic CAFs compared to fibroblasts from nonneoplastic tissue, but the clinical or functional relevance was not explored ${ }^{23}$. Here, we show that ADAM12 is associated with the activated stroma, and poor-prognosis mesenchymal molecular subclasses, in PDAC. It is elevated in the serum of PDAC patients and associates with poor outcome. In an exploratory analysis of patients treated with nab-paclitaxel and gemcitabine, circulating ADAM12 levels predicted outcome.

\section{Results}

\section{ADAM12 associates with activated pancreatic cancer} stroma and poor-prognosis molecular subclasses

We identified ADAM12 in a previous screen for stromal targets of tumor-derived $\mathrm{SHH}^{24}$. To confirm that ADAM12 is expressed in human pancreatic cancers, we queried publically available gene expression datasets that contain normal pancreas and pancreatic cancer tissue. $A D A M 12$ was significantly higher expressed in tumor tissue (Fig. 1a and Supplementary Fig. S1a), and high expression of ADAM12 was associated with worse survival following resection (Fig. 1b and Supplementary Fig. $\mathrm{S} 1 \mathrm{~b})^{25,26}$. Microdissected tumor tissue expression data confirmed a predominantly stromal expression of ADAM12 (Fig. 1c) ${ }^{27}$. To further delineate the source of $A D A M 12$ expression, we measured its expression by species-specific qPCR in patient-derived xenografts $(\mathrm{PDXs})^{28}$. Mouse Adam12 expression in stromal host cells was found to be high compared to other wellcharacterized paralogs (Adam10 and -17; Fig. 1d $)^{14}$.

To determine if ADAM12 expression is a hallmark of tumors with highly activated stromal stellate cells and (myo)fibroblasts, its correlation with known markers for such cells was determined by qPCR in bulk tumor tissue (Fig. 1e, and Supplementary Fig. S2a). A strong correlation of $A D A M 12$ was found with secreted protein acidic and cysteine rich (SPARC), $\alpha$-smooth muscle actin (ACTA2), and fibroblast activation protein (FAP). No strong inverse correlations with tumor cellularity were found. Gene set enrichment analysis revealed a significant enrichment of extracellular matrix and stromal pathway signatures in patients with high ADAM12 expression (Supplementary Fig. S2b).

Subclasses of PDAC have been defined at the gene expression level. All current classifications identify a subtype that is characterized by mesenchymal features and increased stromal infiltration ${ }^{29}$. We found that ADAM12 expression associated with both the Collisson et al. quasi-mesenchymal ${ }^{30}$ and the Bailey et al. squamous subtype tumors $^{31}$ (Fig. 1f). Patients clustered with the activated stroma signature from Moffit et al. ${ }^{32}$ also showed high expression of ADAM12 (Fig. 1g). These analyses show that the expression of ADAM12 associates with poor-prognosis mesenchymal subgroups of PDAC.

\section{ADAM12 expression is driven by tumor cell-derived TGF- $\beta$}

Several tumor-derived signals have been identified that shape the stroma by activating the cells that reside in it. For instance, transforming growth factor beta (TGF- $\beta$ ) is a strong activator of CAFs and pancreatic stellate cells (PSCs) during cancer progression ${ }^{33,34}$. To functionally confirm this activation mechanism to drive ADAM12 expression, we treated human stellate cells with TGF- $\beta$ and other ligands known to be involved in tumor-stroma crosstalk. An upregulation of ADAM12 was only apparent in stellate cells treated with TGF- $\beta$ (Fig. 2a).

ADAM12 exists as soluble proteins ${ }^{12}$. These forms can be generated by shedding of the cell-bound protein, but in humans a soluble isoform (ADAM12-S) also exists. To determine if soluble ADAM12 is produced by activated stellate cells, PS- 1 cells were stimulated using TGF- $\beta$ or by coculturing with primary PDAC tumor cells, and ADAM12 was measured by ELISA in the supernatant of these cultures (Fig. 2b). As for the transcript analysis, a strong upregulation of ADAM12 was observed following TGF- $\beta$ dependent activation of stellate cells. Coculture of human stellate cells with primary tumor cells led to a significant upregulation of soluble ADAM12 that could be blocked by the TGF- $\beta$ pathway inhibitor A83-01, confirming that active TGF- $\beta$ ligand is present in these 

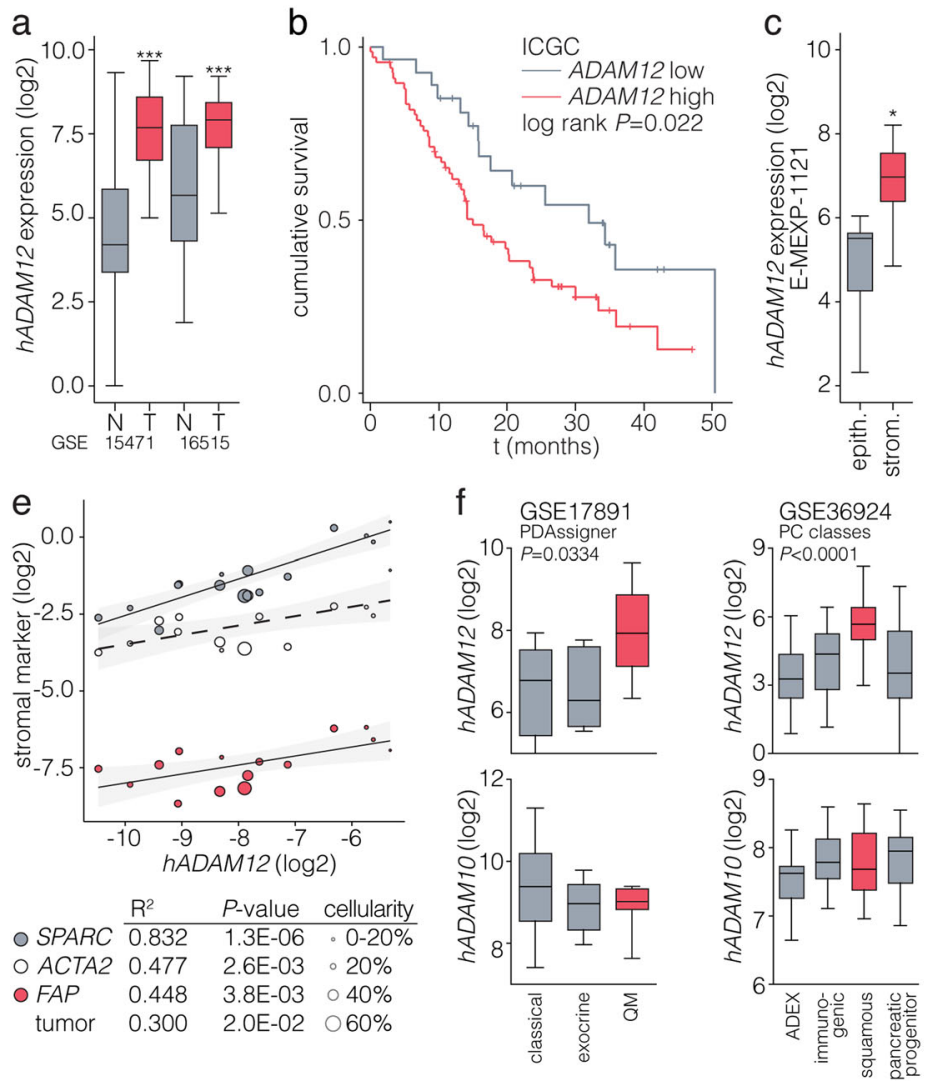
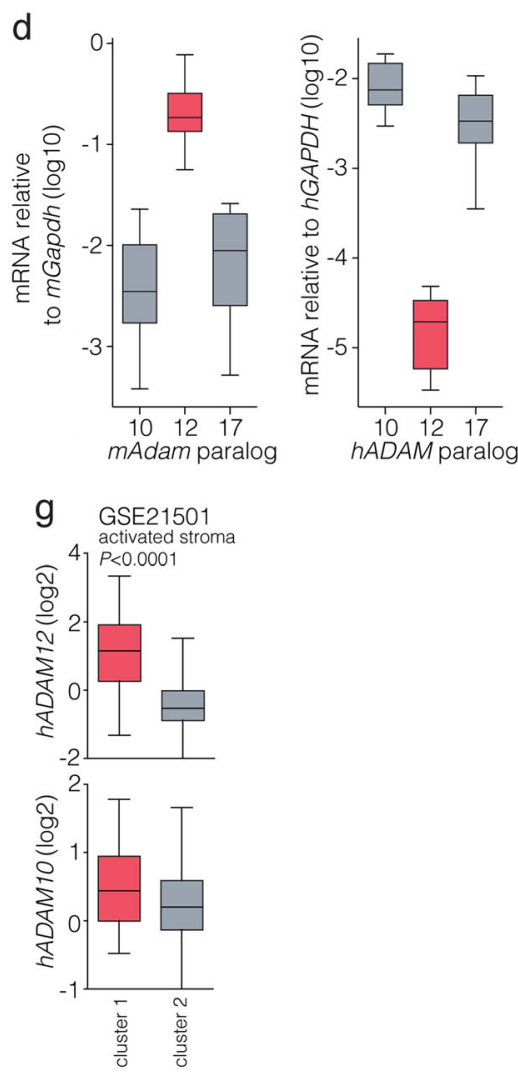

Fig. 1 ADAM12 associates with activated pancreatic cancer stroma and poor-prognosis molecular subclasses. a Boxes indicate median with first and third quartiles of log2 transformed gene expression values from two U133 Plus 2.0 microarray datasets of pancreatic cancer patients comparing normal and tumor tissue. Badea et al. set (GSE15471), $n=36$ paired biopsies; Pei et al. set (GSE16515); $n=16$ (normal), $n=36$ (tumor). ${ }^{* * *} p<0.001$, statistical testing was by two-tailed Student's t test. $\mathbf{b}$ Kaplan-Meier analysis of patients from the ICGC cohort, dichotomized for median ADAM12 expression ${ }^{25}$. c Log2 transformed ADAM12 expression values from the Pilarksy et al. (E-MEXP-1121) ${ }^{27}$ gene expression set obtained from microdissected pancreatic cancer tissue. ${ }^{*} p<0.05$, testing by two-tailed Student's t test. $\mathbf{d}$ Transcript levels for indicated Adam/ADAM paralogs relative to Gapdh/GAPDH were measured in xenografts by qPCR using mouse- or human-specific primers. Boxplots show data from 10 individual patient grafts. For each replicate sample measured by qPCR, a technical triplicate was used. Difference between groups was tested by ANOVA for both panels $p<0.0001$. e Association of ADAM12 expression with stromal activation markers in the AMC patient cohort was measured by qRT-PCR, $n=15$ patients. Size of dots indicates tumor cellularity scored by a pathologist. f Patients from GSE17891 dataset were grouped by published PDAssigner subtype labels and log2 expression of ADAM12 and paralog ADAM10 is shown $(n=27)$. Patients from GSE17891 were grouped by published PC class labels $(n=96)$. Difference between groups was tested by ANOVA. g Patients were k-means clustered using the activated stroma gene set and ADAM12 and -10 expression is shown $(n=132)$. Difference between groups was tested by ANOVA

cocultures and able to drive ADAM12 secretion in stromal cells ${ }^{35}$.

\section{ADAM12 is elevated in the serum of PDAC patients and predicts outcome after resection}

Having established the association of ADAM12 with stromal activation and poor-prognosis molecular subclasses, we proceeded to evaluate ADAM12 as a noninvasive biomarker in PDAC. Patients diagnosed with PDAC before therapeutic intervention showed a significant elevation of serum ADAM12 compared to healthy individuals (Fig. 3a). The association of serum ADAM12 levels with clinical parameters was analyzed (Supplementary Table S1). Patients were dichotomized using serum ADAM12 levels determined by receiver-operatorcharacteristics (ROC, for live-dead resected patients at time of analysis; $316 \mathrm{pg} / \mathrm{ml}$ ). No correlations of serum ADAM12 with age, primary tumor size, and disease stage were found (Supplementary Table S2). High ADAM12 levels in the resected cohort associated with poor survival $(\mathrm{HR}=2.07, p=0.041)$, as did high CA19-9 levels and high LNR (Table 1). In a multivariate analysis no significant associations were found (Supplementary Table S3). The impact of serum ADAM12 on overall survival was analyzed by Kaplan-Meier analysis and log-rank test. We found that whereas serum ADAM12 did not significantly associate with survival in unresectable patients, in resected patients higher ADAM12 levels were strongly 

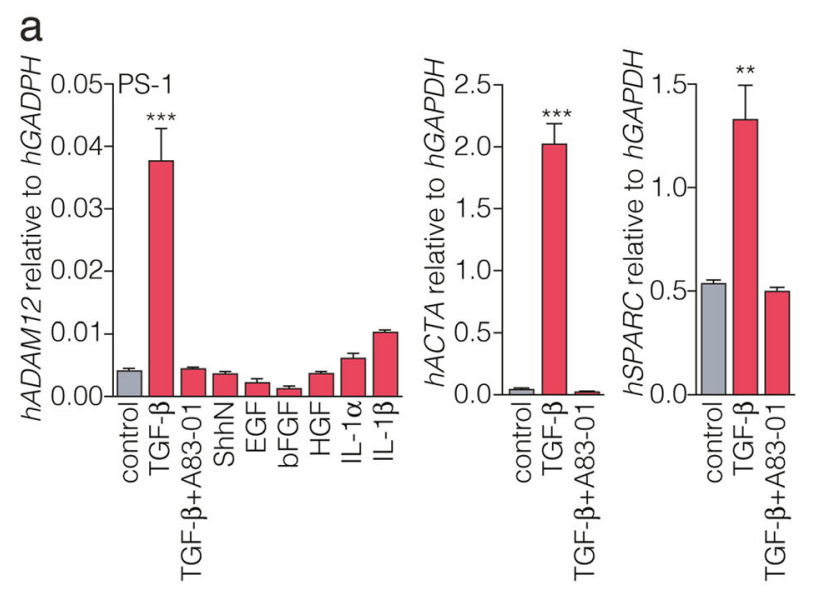

\section{b}

Fig. 2 ADAM12 expression is induced by tumor cell-derived TGF- $\beta$. a Immortalized stellate cells (PS-1 $)^{46}$ were starved with $0.5 \%$ FCS for $24 \mathrm{~h}$ and subsequently treated with the indicated ligands for $48 \mathrm{~h}$. Concentrations used: TGF- $\beta, 5 \mathrm{ng} / \mathrm{mL}$; TGF- $\beta$ receptor I inhibitor A83-01, $1 \mu \mathrm{M}$; ShhN supernatant from $293 \mathrm{~T}$ cells, 1:4; EGF, $50 \mathrm{ng} / \mathrm{ml}$; bFGF, $10 \mathrm{ng} / \mathrm{ml}$; HGF, $10 \mathrm{ng} / \mathrm{ml} ; \mathrm{IL}-1 \mathrm{a}, 10 \mathrm{ng} / \mathrm{ml} ; \mathrm{IL}-1 \beta, 10 \mathrm{ng} / \mathrm{ml}$. In addition to ADAM12, expression analysis of stromal activation marker genes ACTA and SPARC in response to TGF- $\beta$ is shown. Two-tailed Student's $t$ test comparing control vs. TGF- $\beta$, ${ }^{* * *} p<0.001,{ }^{* *} p<0.001 . n=6$, across two separate experiments for control and TGF- $b, n=3$ in one experiment for other treatments. For each replicate sample measured by $\mathrm{qPCR}$, a technical triplicate was used. Bars show mean \pm SEM. $\mathbf{b}$ To ascertain that tumor cell-derived TGF- $\beta$ drives ADAM12 expression, stellate cells were treated as for panel $\mathbf{a}$, or cocultured with tumor cells of the 84 primary culture. Supernatant was harvested and cleared by centrifugation. Soluble ADAM12 levels were measured by ELISA. At least three replicates are shown, statistical testing was by twotailed Student's t test comparing control vs. TGF- $\beta$, and control vs. coculture, ${ }^{* * *} p<0.001 . n=6$, across two separate experiments for treatments, $n=$ 3 in one experiment for coculture. For each replicate sample measured by $\mathrm{PPCR}$, a technical triplicate was used. Bars show mean \pm SEM
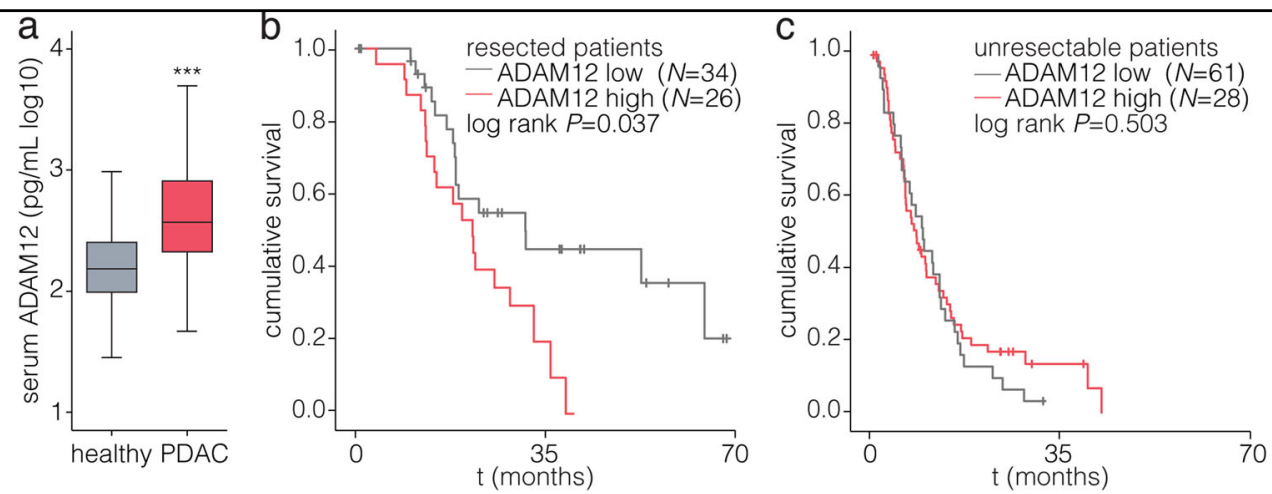

Fig. 3 ADAM12 is elevated in the serum of PDAC patients and predicts poor outcome in patients undergoing resection. a ADAM12 levels were measured by ELISA in serum of healthy individuals $(n=38)$, and patients diagnosed with PDAC $(n=157)$. Boxplots show median with interquartile range. ${ }^{* *} p<0.0001$; tested by Mann-Whitney U-test against healthy controls. $\mathbf{b}$ Kaplan-Meier survival analysis of AMC PDAC patients who underwent resection of the tumor, dichotomized by baseline serum levels determined by receiver-operator-characteristics (ROC; $316 \mathrm{pg} / \mathrm{mL}$ ). $\mathbf{c}$ As for panel $\mathbf{b}$, for patients who did not undergo resection. Statistical testing was by log-rank test

associated with shorter survival (Fig. 3b, c). It thus appears that activated stroma, as revealed by high serum ADAM12 levels, contributes to poor disease outcome when the tumor is at a resectable stage.

\section{ADAM12 levels predict favorable outcome in patients treated with nab-paclitaxel}

The phase III MPACT trial showed survival benefit of nab-paclitaxel with gemcitabine compared to gemcitabine in metastasized PDAC patients, and is relatively well tolerated. To determine if ADAM12 levels associate with response to nab-paclitaxel, we measured its levels in plasma samples from the MPACT cohort $^{9,36,37}$.

Baseline samples were measured and it was observed that the decreased sensitivity of detection in plasma (rather than serum) resulted in a considerable number of samples that had undetectable levels of ADAM12 as defined by $2 \times$ standard deviation of the optical density of 
Table 1 Cox proportional hazard regression model

\begin{tabular}{|c|c|c|c|c|c|c|c|}
\hline \multirow[t]{2}{*}{ Univariate } & & \multicolumn{3}{|c|}{ Resected } & \multicolumn{3}{|c|}{ Unresectable } \\
\hline & & $H R$ & $95 \% \mathrm{Cl}$ & $p$-value & $H R$ & $95 \% \mathrm{Cl}$ & $p$-value \\
\hline Age & & 1.01 & $0.97-1.04$ & 0.73 & 1.02 & $0.99-1.05$ & 0.203 \\
\hline \multirow[t]{2}{*}{ Gender } & Male & 1 & & & 1 & & \\
\hline & Female & 0.64 & $0.31-1.32$ & 0.225 & 1.32 & $0.84-2.07$ & 0.236 \\
\hline \multirow[t]{4}{*}{ Stage } & I & 1 & & & & & \\
\hline & $\|$ & 2.37 & $0.56-10.01$ & 0.242 & 1 & & 0.022 \\
\hline & III & 2.14 & $0.41-11.12$ & 0.368 & 1.44 & $0.59-3.54$ & 0.424 \\
\hline & IV & 3.86 & $0.35-42.93$ & 0.273 & 2.51 & $1.06-5.99$ & 0.038 \\
\hline \multirow[t]{2}{*}{ Tumor size } & $\leq 20 \mathrm{~mm}$ & 1 & & & 1 & & \\
\hline & $>20 \mathrm{~mm}$ & 2.01 & $0.83-4.92$ & 0.124 & 25.36 & $0.37-1749$ & 0.134 \\
\hline \multirow[t]{2}{*}{ CA19-9 } & Low & 1 & & & 1 & & \\
\hline & High & 3.28 & $1.05-10.19$ & 0.04 & 2.11 & $1.21-3.69$ & 0.008 \\
\hline LNR & & 7.49 & $1.61-34.89$ & 0.01 & $\mathrm{n} / \mathrm{a}$ & $\mathrm{n} / \mathrm{a}$ & $\mathrm{n} / \mathrm{a}$ \\
\hline \multirow[t]{2}{*}{ ADAM12 } & Low & 1 & & & 1 & & \\
\hline & High & 2.07 & $1.03-4.16$ & 0.041 & 0.67 & $0.41-1.10$ & 0.116 \\
\hline
\end{tabular}

Univariate analysis for overall survival in PDAC patients (resected $N=58$; unresectable $N=86$ ). Dichotomization of resected patients by CA19-9 was at $415 \mathrm{kU} / \mathrm{L}$, by ADAM12 at $316 \mathrm{pg} / \mathrm{mL}$. Dichotomization of unresectable patients by CA19-9 was at $354 \mathrm{kU} / \mathrm{L}$. LNR lymph node ratio

blanks. Dichotomization of the MPACT cohort by this cutoff resulted in groups with similar size across treatment arms and baseline characteristics (Supplementary Table S4 and S5) but significantly worse survival for patients with detectable ADAM12 (Supplementary Fig. S3a). Univariate Cox regression revealed a HR of 1.41 (1.10-1.81 95\% CI; $p=0.0062)$ for detectable ADAM12 (Supplementary Table S6) in this cohort.

When the trial arms were analyzed separately, ADAM12 levels did not significantly associate with survival in patients that received gemcitabine monotherapy (Fig. 4a, dashed lines, $p=0.2543$ ). Conversely, in patients that received nab-paclitaxel with gemcitabine, undetectable plasma ADAM12 strongly associated with favorable outcome (solid lines, $p=0.0046$ ). Patients with undetectable ADAM12 showed a median survival benefit of over 4.0 months from the addition of nab-paclitaxel to gemcitabine, as compared to a benefit of 1.9 months for patients with detectable ADAM12. Baseline ADAM12 levels were significantly associated with outcome in a multivariate model including KPS, and treatment as factors (Supplementary Table S7). Inclusion of CA19.9 in the model yielded a non-significant association of ADAM12 with survival.

Next, we determined the predictive power of the change in ADAM12 levels during treatment from cycle 1, day 1 (baseline) to cycle 2, day 1 (follow-up) samples (Supplementary Table S4b, baseline characteristics in
Supplementary Table S5b). A reduction in ADAM12 levels is likely to be caused by a diminished stromal activation, or a reduced tumor load. Indeed, a reduction in plasma ADAM12 associated with improved survival (Supplementary Fig. S3b). Importantly, these associations were all driven by the favorable outcome of patients with reduced (OS $11.2 \mathrm{~m}$ ) or repeatedly undetectable (OS $14.4 \mathrm{~m})$ ADAM12 in the nab-paclitaxel with gemcitabinetreated trial arm, as compared to the increased levels (OS 8.3) of ADAM12 (Fig. 4b, c). Although statistical significance was not reached in the gemcitabine arm, the rank ordering of the groups defined by change in ADAM12 levels on treatment was the same in both arms of the trial. In the poorest-outcome group (in which ADAM12 became detectable or increased at cycle 2), the overall survival was numerically superior at 8.3 months in the nab-paclitaxel with gemcitabine arm as compared to the monotherapy arm at 6.9 months. Thus, our results do not indicate an absence of treatment benefit from nabpaclitaxel in the patients with high or increased ADAM12.

In conclusion, we established that ADAM12 is a serumborne proxy for the stromal activation of pancreatic cancers, and that its levels associate with poor disease outcome. A low level of or decrease in ADAM12 is associated with improved survival in patients treated with nab-paclitaxel and gemcitabine, and could possibly be used to stratify patients in future trials using this or other treatment combinations. 
a

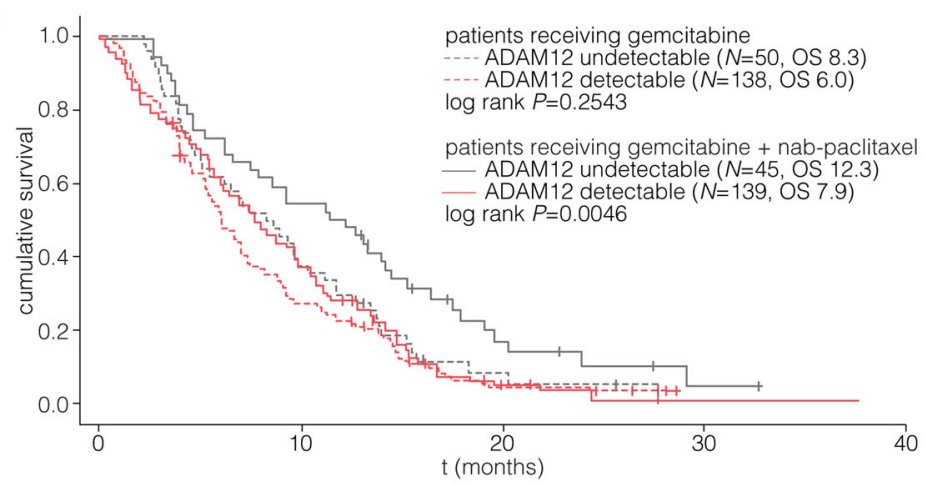

b

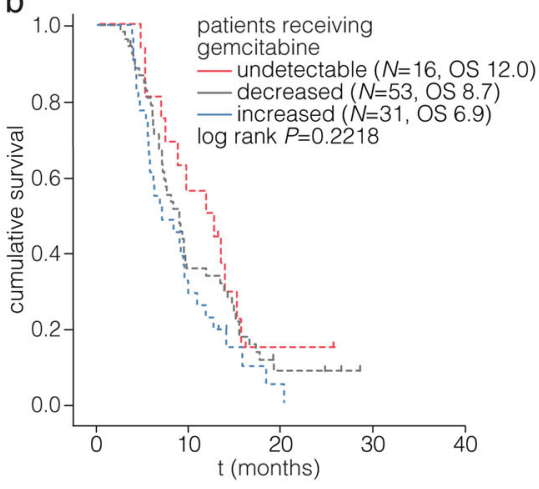

C

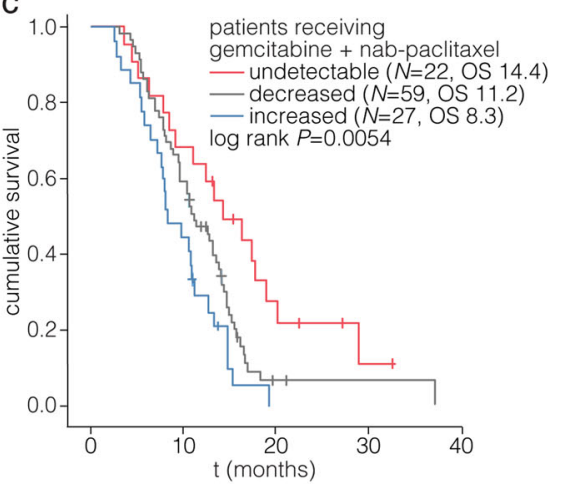

Fig. 4 Plasma ADAM12 predicts favorable outcome in patients treated with nab-paclitaxel and gemcitabine. a Baseline recalcified plasma samples from the MPACT cohort were analyzed for ADAM12 and patients were dichotomized for ADAM12 levels above or below detection ( $2 \times$ SD of blanks). Patients in the arm receiving gemcitabine monotherapy are indicated by dashed lines. Numbers in parentheses indicate number of patients in the group (n), and median survival for that group (OS). Analysis of patients in the arm receiving gemcitabine and nab-paclitaxel is shown with solid lines. b Baseline (cycle 1, day 1) and follow-up (cycle 2, day 1) samples were measured and fold change was calculated. Samples with undetectable ADAM12 levels at both baseline and follow-up did not show a fold change and are indicated by the red line. Patients in the arm receiving gemcitabine monotherapy are shown. $\mathbf{c}$ As for panel $\mathbf{b}$, analysis of patients in the arm receiving gemcitabine and nab-paclitaxel. Statistical testing in all panels was by log-rank test

\section{Discussion}

PDAC is characterized by an abundance of activated stroma that harbors both tumor promoting, as well as suppressive properties ${ }^{38-40}$. Here, analysis of gene expression data, patient-derived xenografts and co-culture experiments identified ADAM12 as a circulating marker for activated stroma and tumors of poor-prognosis molecular subclasses. In patients with resectable disease, high ADAM12 levels predicted inferior survival outcomes. This could imply that ADAM12 is not a general stromal activation marker, but that there are very specific biological programs affected by this protein. Known roles for stromal ADAM12 are manifold: the well characterized function of ADAM12 as a sheddase of growth factor receptor ligands such as EGF can provide a nurturing environment for tumor cells ${ }^{41,42}$. Furthermore, ADAM12 has been shown to participate in matrix remodeling by cleavage of extracellular matrix proteins, suggesting involvement in cancer invasion and metastasis formation $^{21}$. Further studies, similar to ones performed for breast and prostate cancer, are needed to elucidate the functional contributions of ADAM12 to pancreatic cancer.

In metastatic PDAC patients treated with gemcitabine, ADAM12 was not associated with survival. However, low ADAM12 levels did predict response to treatment in the cohort of patients treated with nab-paclitaxel and gemcitabine. This suggests that the supportive role of the stroma in the metastases predominates, to induce resistance to cytotoxic treatment. In this case, low ADAM12 levels identify lesions that are more sensitive to cytotoxic treatment. Clearly, such an effect becomes more apparent in effective treatment regimens, such as nab-paclitaxel with gemcitabine. Future studies are needed to show that ADAM12 retains its predictive power for other effective cytotoxic treatment regimens, such as FOLFIRINOX ${ }^{43}$.

Alternatively, it could be argued that the stromatargeting properties of nab-paclitaxel explain why low ADAM12 predicts favorable outcome of nab-paclitaxel and gemcitabine. Nab-paclitaxel is accepted to have at 
least some degree of specificity for the stroma: the conjugation to albumin has been suspected to increase taxane concentration at sites of high SPARC (an albumin receptor) expression such as stromal (myo)fibroblasts ${ }^{6}$. In tumors with relatively few activated stromal cells, nabpaclitaxel will therefore be able to effectively eradicate the vast majority of the stroma, whereas in tumors with highly abundant stroma, the ablation is only partial and not effective in the long term.

One limitation of the current study is the exploratory, retrospective nature of this analysis. The MPACT trial was not statistically powered to test the interaction between serum markers such as ADAM12 and treatment groups. Furthermore, it is possible that the predictive value of ADAM12 as inferred from the differences in response to treatment is a result of the longer survival of patients receiving nab-paclitaxel with gemcitabine. Moreover, it should be noted that other biomarker discovery studies have not yet yielded applicable markers. For instance the initially suspected role for SPARC in conferring enhanced sensitivity to nab-paclitaxel in PDAC was not confirmed ${ }^{7,8}$, and its expression did not correlate with response to nab-paclitaxel with gemcitabine. Decreased CA19-9 levels were initially described to associate with response rate in the experimental arm of the MPACT trial ${ }^{37}$, and high baseline CA19.9 was associated with reduction in risk of death from the combination treatment (gemcitabine with nab-paclitaxel) ${ }^{9}$. However, in a follow-up analysis CA19-9 levels did not predict survival in the combination treatment $\mathrm{arm}^{36}$. Other studies have identified panels of serum-borne markers for PDAC stroma and correlated these to response in experimental models for, but so far, predictive value has not been assessed ${ }^{11}$.

Although the significant association of serum ADAM12 with outcome was not sustained in multivariate analysis of the MPACT samples when CA19-9 was included in our model, levels of ADAM12 were very strongly associated with favorable responses to nab-paclitaxel with gemcitabine suggesting that the analysis of a sufficiently large cohort could confirm predictive power. This will offer important possibilities for patient stratification. ADAM12, or similar blood-borne proxies for stromal activation could possibly be used to stratify patients in future trials with experimental stroma targeting agents, as well as for currently used (chemo)therapeutics. In summary, we have shown that ADAM12 expression in pancreatic cancer is indicative of highly activated stroma as well as poorprognosis mesenchymal subclasses of tumors. Systemic levels of ADAM12 were found to be strongly prognostic in resectable PDAC patients, and low ADAM12 levels were associated with improved survival in patients treated with nab-paclitaxel and gemcitabine. ADAM12, or similar blood-borne proxies for stromal activation could possibly be used to stratify patients in future trials with experimental stroma targeting agents, as well as for currently used (chemo)therapeutics. To definitively establish the predictive power of ADAM12 or similar proxies for stromal activation in solid cancers, we propose a prospective clinical trial, using the levels of such markeres as intervention to stratify patients for currently available (chemo)therapeutics, but also experimental stroma targeting agents.

\section{Materials and methods \\ Collection of blood samples}

In the AMC cohort, serum samples were obtained perioperatively from 60 patients undergoing resection, or before the start of treatment in case of unresectable patients $(n=89)$. Clinicopathological data were obtained from medical records and included age, gender, tumor diameter, differentiation grade, lymph node ratio (positive lymph nodes/total number of lymph nodes examined), therapies received, and tumor-node-metastasis (TNM) staging. Collection of material was approved by the institute's ethics committee (IRB), and written informed consent was received from all participants (AMC METC $\left.2014 \_181\right)^{28}$. Blood samples of 38 non-age matched healthy individuals without any indication of malignancy were collected as a control group. In the MPACT trial cohort, collection of plasma samples for biomarker development was optional and separate written consent was obtained for sample collection and biomarker analysis. The patients and methods of the MPACT trial have been described previously (trial registration number NCT00844649) ${ }^{37}$. All patients provided infromed consent. In brief, patients were $\geq 18$ years of age, had confirmed measurable metastatic adenocarcinoma of the pancreas, a Karnofsky performance status (KPS) of $\geq 70$, and did not receive prior chemotherapy for metastatic disease. Patients were randomized 1:1 (stratified by performance status, presence of liver metastases, and region) to receive $n a b$-paclitaxel $125 \mathrm{mg} / \mathrm{m}^{2}$ plus gemcitabine $1000 \mathrm{mg} / \mathrm{m}^{2}$ on days 1,8 , and 15 of every 28 days or gemcitabine alone $1000 \mathrm{mg} / \mathrm{m}^{2}$ on days $1,8,15,22,29,36$, and 43 in 56 days (cycle 1 ) and then on days 1,8 , and 15 of every 28 days (cycle $\geq 2$ ). Treatment continued until disease progression or unacceptable toxicity.

\section{Establishment of patient-derived xenografts and primary cell lines}

Collection of tumor material was approved by the institute's ethics committee in accordance with the Declaration of Helsinki (AMC METC 2014_181). Written informed consent was received from all participants ${ }^{28}$. All PDAC patients treated in the Academic Medical Center were diagnosed by pathology or cytology. Specimens were processed and inspected according to national and 
international guidelines. An experienced pathologist performed microscopic assessment. Final diagnosis was set in accordance with the WHO classification, and the pTNM classification of malignant tumors. Freshly excised tumor pieces (approximately $3 \times 3 \times 3 \mathrm{~mm}$ ) originating from the primary tumor or liver metastasis were washed several times in PBS containing $10 \mu \mathrm{g} / \mathrm{ml}$ gentamycin (Lonza, Basel, Switzerland) and 1\% penicillin-streptamycin. Pieces were grafted subcutaneously into the flank of immunocompromised NOD.Cg-Prkdc ${ }^{\text {scid }}$ Il $2 \mathrm{rg}^{\mathrm{tm} 1 \mathrm{~W}_{\mathrm{jl}}} / \mathrm{SzJ}$ (NSG) mice (JAX 005557) with Matrigel (BD, Franklin Lakes, $\mathrm{NJ})$. Both sexes were used, and ages ranged up to 9 months. Animals were bred and maintained at the local animal facility according to the pertinent legislation, and ethical approval was obtained for all procedures (DTB/ LEX102348). After outgrowth to a size of $\sim 500 \mathrm{~mm}^{3}$, tumors were harvested and passaged, and/or used to establish in vitro cultures. For this, harvested xenografts were minced with a scalpel blade, placed in IMDM with $8 \%$ FBS and collagenase IV $(0.5 \mathrm{mg} / \mathrm{ml}$, Sigma-Aldrich, St. Louis, MO) and incubated at $37^{\circ} \mathrm{C}$ for 60 min with vortexing every $15 \mathrm{~min}$. The dissociated suspension was passed through a $70 \mu \mathrm{m}$ cell strainer, washed with culture medium and grown in IMDM containing $8 \%$ FBS and $50 \mu \mathrm{M} \beta$-mercaptoethanol. During the first 5-10 passages, cultures contained colonies of human epithelial cells and a layer of murine fibroblasts. A culture without epithelial component, as confirmed by flow cytometry using an anti-EPCAM antibody (DAKO, F0860 at 1:100), was used for stimulation experiments. STR profiling was performed to confirm donor-cell line matching (August 2017). For transcript analysis, all eligible and available xenografts were analyzed. Randomization and blinding did not apply. Xenografts were excluded if histopathological assessment did not confirm diagnosis of PDAC.

\section{RNA isolation and quantitative real-time PCR}

Small pieces of PDX tumor were homogenized using an Ultra-Turrax tissue homogenizer T8 (IKA-Werke, Staufen im Breisgau, Germany) in $1 \mathrm{ml}$ of Trizol (ThermoFisher). Primary cells were lysed in Trizol and RNA isolation was performed according to the manufacturer's protocol. Snap frozen patient tumor samples were embedded in Tissue-Tek OCT (Sakura FineTek, Japan) and 30 slices of $20 \mu \mathrm{m}$ each were cut on a cryotome. Cut tissue was immersed in $1 \mathrm{ml}$ of RNA Bee (Amsbio, Abingdon, United Kingdom), homogenized, and RNA isolation was performed according to manufacturer's protocol (Qiagen, Hilden, Germany). For tumor percentage scoring, a $10 \mu \mathrm{m}$ slice was kept before the tissue was cut in $20 \mu \mathrm{m}$ slices, and processed for H\&E staining. Scoring of tumor percentage was performed by an experienced pathologist. cDNA was synthesized using Superscript III (ThermoFisher) and random primers. Real-time quantitative RT-PCR was performed with SYBR green (Roche, Basel, Switzerland) on a Lightcycler LC480 II (Roche). Relative expression of genes was calculated using the comparative threshold cycle $(\mathrm{Cp})$ method and values were normalized to reference gene GAPDH/ Gapdh. Primer sequences are: $h G A P D H$ Fw $5^{\prime}$ gaaggtgaaggtcggagtc $3^{\prime}$; $h G A P D H$ Rv $5^{\prime}$ tggaagatggtgatgggatt 3'; hADAM10 Fw $5^{\prime}$ ttcgatgcaaatcaaccaga $3^{\prime} ;$ hADAM10 Rv $5^{\prime}$ ttccttccettgcacagtct $3^{\prime}$; hADAM12 Fw $5^{\prime}$ tttccaccaccctctcagac $3^{\prime} ;$ hADAM12 Rv 5' gcctctgaaactctcggttg 3'; hADAM17 Fw 5' gggaacatgaggcagtctct $3^{\prime} ;$ hADAM17 Rv $5^{\prime}$ accgaatgctgctggatatt $3^{\prime} ;$ hACTA2 $\mathrm{Fw} 5^{\prime}$ caaagccggccttacagag $3^{\prime} ;$ hACTA2 Rv 5' agcccagccaagcactg $3^{\prime} ;$ hFAP Fw $5^{\prime} \quad$ tcagtgtgagtgctctcattgtat $3^{\prime} ; \quad h F A P \quad \mathrm{Rv} \quad 5^{\prime}$ gctgtgcttgccttattggt $3^{\prime} ; \quad h S P A R C \quad \mathrm{Fw} \quad 5^{\prime}$ gaaagaagatccaggccctc $3^{\prime} ;$ hSPARC Rv 5' cttcagactgcccggaga 3'; hKRT19 Fw 5' cctggagttctcaatggtgg 3'; hKRT19 Rv 5' ctagaggtgaagatccgcga $3^{\prime}$; mGapdh $\mathrm{Fw} 5^{\prime}$ ctcatgaccacagtccatgc 3'; mGapdh Rv 5' cacattgggggtaggaacac 3'; mAdam10 Fw 5' aagatggtgttgccgacagt $3^{\prime} ;$ Adam10 Rv 5' tggtcctcatgtgagactgc $3^{\prime}$; mAdam12 Fw $5^{\prime}$ gctttggaggaagcacagac $3^{\prime} ;$ mAdam12 $\mathrm{Rv} 5^{\prime}$ cgcatcaacgtcttcctttt 3'; mAdam17 Fw 5' gtacagcgtgaagtggcaga 3'; mAdam17 Rv $5^{\prime}$ gccccatctgtgttgattct $3^{\prime}$.

\section{Gene set enrichment analysis (GSEA) and expression analysis}

Gene set enrichment analysis (v2.0.14) software was downloaded from the Broad Institute website (http:// www.broadinstitute.org/gsea) and used according to the author's guidelines ${ }^{44}$. Median ADAM12 expression was used to dichotomize samples. Gene set for the GO term 'extracellular matrix' was downloaded from the Molecular Signature Database (MSigDB; V4.0); the pancreatic stroma signature was published by Binkley et al.$^{45} 2000$ phenotype permutations were used to determine significance of the enrichment score. Gene expression data were collected and processed for use in the AMC inhouse R2 Genomics Analysis and Visualization Platform: (http://r2.amc.nl). For visualization of gene expression, data were imported in $\mathrm{R}$ and plotted using ggplot2, or plotted in Graphpad Prism.

\section{Treatment of cultures}

Stellate cells (from the Kocher $1 \mathrm{lab}^{46}$ ) were seeded in 12well culture plates and upon reaching confluence, prestarved overnight in $0.5 \%$ FBS containing medium and treated for $24 \mathrm{~h}$ with $5 \mathrm{ng} / \mathrm{ml}$ recombinant human TGF$\beta 1$ (Peprotech, London, UK) in the presence or absence of $1 \mu \mathrm{M}$ ALK4/5/7 inhibitor A83-01 (Tocris Bioscience, 
Bristol, UK). ShhN supernatant from 293T cells was added 1:4, EGF was used at $50 \mathrm{ng} / \mathrm{ml}$, bFGF, $10 \mathrm{ng} / \mathrm{ml}$; HGF $10 \mathrm{ng} / \mathrm{ml} ; \mathrm{IL}-1 \alpha, 10 \mathrm{ng} / \mathrm{ml}$; IL- $1 \beta, 10 \mathrm{ng} / \mathrm{ml}$. Treatment was not randomized between plate wells, and experiments were not blinded. Cultures were STR authenticated (June 2016), and tested for mycoplasma monthly.

\section{ELISA analysis of serum samples (AMC cohort)}

For the reporting on association of ADAM12 levels with prognosis, the pertinent guidelines were considered $^{47}$. Serum was obtained by centrifugation of blood for $10 \mathrm{~min}$ at room temperature at $1300 \times \mathrm{g}$, and storage at $-80^{\circ} \mathrm{C}$ until analysis. ADAM12 levels were determined using the human ADAM12 DuoSet ELISA kit (R\&D Systems, Minneapolis, MN), according to manufacturer's recommendations. Briefly, after coating the 96-well plates (Greiner Nunc MaxiSorp, Kremsmünster, Austria) with capture antibody overnight at room temperature, blocking the plate with $1 \%$ BSA the following day, $80 \mu$ of serum samples were added. After incubation for two hours at room temperature and mild washing steps, samples were incubated with biotinylated detection antibody for additional two hours followed by a $20 \mathrm{~min}$ incubation step with horse-radish peroxidase (HRP)-labeled streptavidin. Substrate was tetramethylbenzidine substrate solution (TMB), added for an additional $20 \mathrm{~min}$. Absorbance was measured at $450 \mathrm{~nm}$ and $570 \mathrm{~nm}$ with a microplate reader (BioTek Synergy BioTek, Winooski, VT) after addition of $1 \mathrm{M} \mathrm{H}_{2} \mathrm{SO}_{4}$ stop solution. For wavelength correction, $570 \mathrm{~nm}$ readings were subtracted from the $450 \mathrm{~nm}$ values before further analysis. The technician performing the analysis was blinded for survival time. Randomization did not apply.

\section{ELISA analysis of plasma samples (MPACT cohort)}

MPACT trial blood samples were collected in EDTA tubes to chelate calcium and prevent blood clotting, and storage at $-80^{\circ} \mathrm{C}$ until analysis. To allow the analysis of these plasma samples using the ADAM12 DuoSet ELISA, recalcification was required. Hundred microliters of EDTA plasma was complemented with $12 \mathrm{mM} \mathrm{CaCl}_{2}$ (final concentration) and incubated overnight at $4{ }^{\circ} \mathrm{C}$. The following day, clots were removed manually and $50 \mu \mathrm{l}$ sample was used for the assay. The ELISA was performed as for the serum samples. Analysis was blinded to the technician performing the ELISA, who was unaware of the trial arm the samples were from. Sensitivity of the assay was reduced compared to measurement in serum by a factor of 0.44, explaining why in the MPACT cohort, the lowest quartile was composed of samples that had undetectable plasma ADAM12. Measurement of ADAM12 in matched serum and plasma samples revealed high correlation $\left(\mathrm{R}^{2}=0.9971, p\right.$-value: $\left.2.0 \times 10^{-6}, n=6\right)$.

\section{Statistics}

Student's t-tests were performed using GraphPad Prism 5 software (GraphPad, La Jolla, CA). R was used for linear regression analysis of gene expression. All tests were twosided and $p<0.05$ was considered statistically significant. Variance was assumed to be similar between compared groups. Adjustments for multiple comparisons were applied for gene correlations across the genome (FDR). For the cell culture experiments, no sample size calculation was performed. For the AMC cohort patient data, SPSS package 24. (IBM Analytics, Armonk, NY) was used for Chi-square testing, Mann-Whitney U test, Spearman's rank correlation, Cox proportional hazard regression modeling, Kaplan-Meier survival analysis, and logrank test. Patients who died within 30 days after operation were excluded from survival analysis as these likely did not succumb to cancer $(n=5)$. Otherwise, all eligible samples were measured and no power calculation was performed. Cox proportional hazard regression was used for univariate and multivariate analyses to investigate the correlation of OS with ADAM12 and potential prognostic factors.

For the MPACT cohort, baseline ADAM12 values were categorized into undetectable $(0)$ and detectable $(>0)$. The fold change of ADAM12 at cycle 2, day 1 (C2D1) from baseline (cycle 1, day 1; C1D1) was calculated by the results at $\mathrm{C} 2 \mathrm{D} 1$ divided by the value at $\mathrm{C} 1 \mathrm{D} 1$. These were then assigned to three groups (0: both values below detection limit; $<1$ if levels decreased, $>1$ if values increased). Descriptive statistics summary of demographics and baseline characteristics were performed. Correlation of ADAM12 groups with categorical variables was tested using $\mathrm{CMH}$ statistics or Cochran-Armitage Trend Test statistics, or one-way ANOVA for continuous variables. All eligible samples were measured and no power calculation was performed. Overall survival was analyzed using Kaplan-Meier method and log-rank test. SAS 9.2 (SAS Institute Inc., Cary, NC) was used for all statistical analyses in the MPACT study.

\footnotetext{
Acknowledgements

We thank Tom van Leusden for technical assistance, and all patients for participating. This work was supported by KWF Dutch Cancer Society (UVA 2012-5607 and UVA 2013-5932).

\section{Author details}

${ }^{1}$ Amsterdam UMC, University of Amsterdam, LEXOR, Center for Experimental and Molecular Medicine, Cancer Center Amsterdam, Amsterdam, Netherlands. ${ }^{2}$ Oncode Institute, Meibergdreef 9, 1105AZ Amsterdam, Netherlands.

${ }^{3}$ Amsterdam UMC, University of Amsterdam, Department of Surgery, Cancer Center Amsterdam, Amsterdam, Netherlands. ${ }^{4}$ Amsterdam UMC, University of Amsterdam, Department of Pathology, Cancer Center Amsterdam, Amsterdam, Netherlands. ${ }^{5}$ Amsterdam UMC, University of Amsterdam, Department of Medical Oncology, Cancer Center Amsterdam, Amsterdam, Netherlands. ${ }^{6}$ Wolfson Wohl Cancer Research Centre, Institute of Cancer Sciences, University of Glasgow, Glasgow, UK. ${ }^{7}$ West of Scotland Pancreatic Unit, Glasgow Royal Infirmary, Glasgow, UK. ${ }^{8}$ Centre for Tumour Biology, Barts Cancer Institute, Queen Mary University of London, London, UK. ${ }^{9}$ Celgene Corporation, Summit,
} 
NJ, USA. ${ }^{10}$ Present address: Biotech Research and Innovation Centre, University of Copenhagen, Copenhagen, Denmark

\section{Conflict of interest}

M.F.B. has received research funding from Celgene. H.W.L. has acted as a consultant for Celgene, Eli Lilly and Company, Nordic Pharma Group and Philips, has received research grants from, Amgen, Bayer Schering Pharma AG, Celgene, Eli Lilly and Company, GlaxoSmithKline Pharmaceuticals, Nordic Pharma Group, Philips, Roche Pharmaceuticals. D.W.P., R.J., and J.S.L. are Celgene employees. Other than Celgene, none were involved in drafting of the manuscript.

\section{Publisher's note}

Springer Nature remains neutral with regard to jurisdictional claims in published maps and institutional affiliations.

Supplementary Information accompanies this paper at (https://doi.org/ 10.1038/s41389-018-0096-9)

Received: 24 August 2018 Revised: 9 October 2018 Accepted: 24 October 2018

\section{Published online: 16 November 2018}

\section{References}

1. Rahib, L. et al. Projecting cancer incidence and deaths to 2030: the unexpected burden of thyroid, liver, and pancreas cancers in the United States. Cancer Res. 74, 2913-2921 (2014).

2. Hidalgo, M. Pancreatic cancer. N. Engl. J. Med. 362, 1605-1617 (2010).

3. Bachem, M. G. et al. Pancreatic carcinoma cells induce fibrosis by stimulating proliferation and matrix synthesis of stellate cells. Gastroenterology $\mathbf{1 2 8}$, 907-921 (2005)

4. Whatcott, C., Han, H., Posner, R. G. \& Von Hoff, D. D. Tumor-stromal interactions in pancreatic cancer. Crit. Rev. Oncog. 18, 135-151 (2013).

5. Alvarez, R. et al. Stromal disrupting effects of nab-paclitaxel in pancreatic cancer. Br. J. Cancer 109, 926-933 (2013).

6. Desai, N. Trieu, V., Damascelli, B. \& Soon-Shiong, P. SPARC expression correlates with tumor response to albumin-bound paclitaxel in head and neck cancer patients. Transl. Oncol. 2, 59-64 (2009).

7. Hidalgo, M. et al. SPARC expression did not predict efficacy of nab-paclitaxel plus gemcitabine or gemcitabine alone for metastatic pancreatic cancer in an exploratory analysis of the phase III MPACT trial. Clin. Cancer Res. 21, 4811-4818 (2016)

8. Neesse, A. et al. SPARC independent drug delivery and antitumour effects of nab-paclitaxel in genetically engineered mice. Gut 63, 974-983 (2014).

9. Von Hoff, D. D. et al. Increased survival in pancreatic cancer with nab-paclitaxe plus gemcitabine. N. Engl. J. Med. 369, 1691-1703 (2013)

10. Bijlsma, M. F. \& van Laarhoven, H. W. The conflicting roles of tumor stroma in pancreatic cancer and their contribution to the failure of clinical trials: a systematic review and critical appraisal. Cancer Metastas-. Rev. 34, 97-114 (2015).

11. Resovi, A. et al. Soluble stroma-related biomarkers of pancreatic cancer. $E M B O$ Mol. Med 10, e8741 (2018).

12. Kveiborg, M., Albrechtsen, R., Couchman, J. R. \& Wewer, U. M. Cellular roles of ADAM12 in health and disease. Int. J. Biochem. Cell. Biol. 40, 1685-1702 (2008).

13. Asakura, M. et al. Cardiac hypertrophy is inhibited by antagonism of ADAM12 processing of HB-EGF: metalloproteinase inhibitors as a new therapy. Nat. Med. 8, 35-40 (2002)

14. Damhofer, $\mathrm{H}$. et al. Blocking Hedgehog release from pancreatic cancer cells increases paracrine signaling potency. J. Cell Sci 128, 129-139 (2014).

15. Horiuchi, K. et al. Substrate selectivity of epidermal growth factor-receptor ligand sheddases and their regulation by phorbol esters and calcium influx. Mol. Biol. Cell. 18, 176-188 (2007).

16. Frohlich, $\mathrm{C}$. et al. Molecular profiling of ADAM12 in human bladder cancer Clin. Cancer Res.: Off. J. Am. Assoc. Cancer Res. 12, 7359-7368 (2006)

17. Kodama, $\mathrm{T}$. et al. ADAM12 is selectively overexpressed in human glioblastomas and is associated with glioblastoma cell proliferation and shedding of heparin-binding epidermal growth factor. Am. J. Pathol. 165, 1743-1753 (2004).

18. Le Pabic, $H$. et al. ADAM12 in human liver cancers: TGF-beta-regulated expression in stellate cells is associated with matrix remodeling. Hepatology 37, 1056-1066 (2003)

19. Peduto, L. et al. ADAM12 is highly expressed in carcinoma-associated stroma and is required for mouse prostate tumor progression. Oncogene 25, 5462-5466 (2006)

20. Rocks, N. et al. Expression of a disintegrin and metalloprotease (ADAM and ADAMTS) enzymes in human non-small-cell lung carcinomas (NSCLC). Br. J. Cancer 94, 724-730 (2006).

21. Roy, R., Wewer, U. M., Zurakowski, D., Pories, S. E. \& Moses, M. A. ADAM 12 cleaves extracellular matrix proteins and correlates with cancer status and stage. J. Biol. Chem. 279, 51323-51330 (2004).

22. Shao, $\mathrm{S}$. et al. ADAM-12 as a diagnostic marker for the proliferation, migration and invasion in patients with small cell lung cancer. PLoS One 9, e85936 (2014).

23. $Y u, J$. et al. Unlike pancreatic cancer cells pancreatic cancer associated fibroblasts display minimal gene induction after 5-aza-2'-deoxycytidine. PLoS One 7, e43456 (2012).

24. Damhofer, $\mathrm{H}$. et al. Assessment of the stromal contribution to Sonic Hedgehog-dependent pancreatic adenocarcinoma. Mol. Oncol. 7, 1031-1042 (2013).

25. Perez-Mancera, P. A. et al. The deubiquitinase USP9X suppresses pancreatic ductal adenocarcinoma. Nature 486, 266-270 (2012).

26. Zhang, G. et al. Integration of metabolomics and transcriptomics revealed a fatty acid network exerting growth inhibitory effects in human pancreatic cancer. Clin. Cancer Res.: Off. J. Am. Assoc. Cancer Res. 19, 4983-4993 (2013).

27. Pilarsky, C. et al. Activation of Wnt signalling in stroma from pancreatic cancer identified by gene expression profiling. J. Cell. Mol. Med. 12(6B), 2823-2835 (2008).

28. Damhofer, $\mathrm{H}$. et al. Establishment of patient-derived xenograft models and cel lines for malignancies of the upper gastrointestinal tract. J. Transl. Med. 13, 115 (2015).

29. Bijlsma, M. F., Sadanandam, A., Tan, P. \& Vermeulen, L. Molecular subtypes in cancers of the gastrointestinal tract. Nat. Rev. 14, 333-342 (2017).

30. Collisson, E. A. et al. Subtypes of pancreatic ductal adenocarcinoma and their differing responses to therapy. Nat. Med. 17, 500-503 (2011).

31. Bailey, P. et al. Genomic analyses identify molecular subtypes of pancreatic cancer. Nature 531, 47-52 (2016).

32. Moffitt, R. A. et al. Virtual microdissection identifies distinct tumor- and stromaspecific subtypes of pancreatic ductal adenocarcinoma. Nat. Genet. 47 1168-1178 (2015).

33. Apte, M. V. et al. Pancreatic stellate cells are activated by proinflammatory cytokines: implications for pancreatic fibrogenesis. Gut 44, 534-541 (1999).

34. Kordes, C., Brookmann, S., Haussinger, D. \& Klonowski-Stumpe, H. Differential and synergistic effects of platelet-derived growth factor-BB and transforming growth factor-beta1 on activated pancreatic stellate cells. Pancreas 31, 156-167 (2005).

35. Tojo, M. et al. The ALK-5 inhibitor A-83-01 inhibits Smad signaling and epithelial-to-mesenchymal transition by transforming growth factor-beta. Cancer Sci. 96, 791-800 (2005)

36. Goldstein, D. et al. nab-Paclitaxel plus gemcitabine for metastatic pancreatic cancer: long-term survival from a phase III trial. J. Natl. Cancer. Inst 107, dju413 (2015).

37. Von Hoff, D. D. et al. Gemcitabine plus nab-paclitaxel is an active regimen in patients with advanced pancreatic cancer: a phase I/II trial. J. Clin. Oncol.: Off. J. Am. Soc. Clin. Oncol. 29, 4548-4554 (2011).

38. Lee, J. J. et al. Stromal response to Hedgehog signaling restrains pancreatic cancer progression. Proc. Natl Acad. Sci. USA. 111, E3091-E3100 (2014).

39. Ozdemir, B. C. et al. Depletion of carcinoma-associated fibroblasts and fibrosis induces immunosuppression and accelerates pancreas cancer with reduced survival. Cancer Cell. 25, 719-734 (2014).

40. Rhim, A. D. et al. Stromal elements act to restrain, rather than support, pancreatic ductal adenocarcinoma. Cancer Cell. 25, 735-747 (2014).

41. Ardito, C. M. et al. EGF receptor is required for KRAS-induced pancreatic tumorigenesis. Cancer Cell. 22, 304-317 (2012)

42. Yamanaka, $Y$. et al. Coexpression of epidermal growth factor receptor and ligands in human pancreatic cancer is associated with enhanced tumor aggressiveness. Anticancer Res. 13, 565-569 (1993). 
43. Conroy, T. et al. FOLFIRINOX versus gemcitabine for metastatic pancreatic cancer. N. Engl. J. Med. 364, 1817-1825 (2011).

44. Subramanian, A. et al. Gene set enrichment analysis: a knowledge-based approach for interpreting genome-wide expression profiles. Proc. Natl Acad. Sci. USA. 102, 15545-15550 (2005).

45. Binkley, C. E. et al. The molecular basis of pancreatic fibrosis: common stromal gene expression in chronic pancreatitis and pancreatic adenocarcinoma. Pancreas 29, 254-263 (2004).
46. Kadaba, R. et al. Imbalance of desmoplastic stromal cell numbers drives aggressive cancer processes. J. Pathol. 230, 107-117 (2013).

47. MCShane, L. M. et al. Reporting recommendations for tumor marker prognostic studies (REMARK). J. Natl. Cancer Inst. 97, 1180-1184 (2005). 